\title{
Metallomics
}

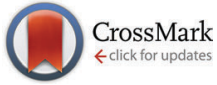

Cite this: Metallomics, 2014, 6,2090

\section{A new insight into the zinc-dependent DNA-cleavage by the colicin E7 nuclease: a crystallographic and computational study $\dagger$}

\author{
Anikó Czene, ${ }^{a}$ Eszter Tóth, ${ }^{b}$ Eszter Németh, ${ }^{\mathrm{b}}$ Harm Otten, ${ }^{\mathrm{c}}$ Jens-Christian N. Poulsen, \\ Hans E. M. Christensen, ${ }^{d}$ Lubomir Rulišek, ${ }^{e}$ Kyosuke Nagata, ${ }^{f}$ Sine Larsen ${ }^{\star c}$ and \\ Béla Gyurcsik*ab
}

\begin{abstract}
The nuclease domain of colicin E7 metallonuclease (NCOIE7) contains its active centre at the C-terminus.

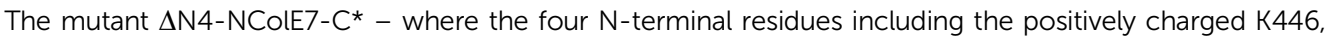
R447 and K449 are replaced with eight residues from the GST tag - is catalytically inactive. The crystal structure of this mutant demonstrates that its overall fold is very similar to that of the native NColE7 structure. This implicates the stabilizing effect of the remaining $\mathrm{N}$-terminal sequence on the structure of the $\mathrm{C}$-terminal catalytic site and the essential role of the deleted residues in the mechanism of the catalyzed reaction. Complementary QM/MM calculations on the protein-DNA complexes support the less favourable cleavage by the mutant protein than by NColE7. Furthermore, a water molecule as a possible ligand for the $\mathrm{Zn}^{2+}$-ion is proposed to play a role in the catalytic process. These results suggest that the mechanism of the $\mathrm{Zn}^{2+}$-containing $\mathrm{HNH}$ nucleases needs to be further studied and discussed.
\end{abstract}

Received 22nd July 2014, Accepted 21st August 2014

DOI: $10.1039 / \mathrm{c} 4 \mathrm{mt} 00195 \mathrm{~h}$

www.rsc.org/metallomics contains conserved $\mathrm{H}$ and $\mathrm{N}$ amino acids in the $\mathrm{HHX}_{14} \mathrm{NX}_{8} \mathrm{HX}_{3} \mathrm{H}$ (amino acids 544-573) pattern. ${ }^{12}$ In this $\beta \beta \alpha$ - metal ion binding stretch $\mathrm{H} 544, \mathrm{H} 569$ and $\mathrm{H} 573$ act as ligands to the $\mathrm{Zn}^{2+}$-ion, ${ }^{7}$ while H545 is supposed to generate the nucleophilic agent by deprotonating a water molecule. ${ }^{9,13,14}$ The catalytic mechanism is well established, with the exception of the protonation of the leaving group. The hypothesized pathways of this step are dependent on the quality of the metal ion in the active centre. The proton may be donated by a water molecule coordinated to a $\mathrm{Ni}^{2+}$-ion in NColE9 ${ }^{15}$ and to a $\mathrm{Mg}^{2+}$-ion in Vvn, ${ }^{16}$ related $\mathrm{HNH}$ nucleases. But for NColE7 the question remains open due to the absence of the corresponding inner-sphere water molecule around the $\mathrm{Zn}^{2+}$-ion. ${ }^{7,17}$ A putative proton channel was suggested in the related colicin E9 nuclease ${ }^{18-20}$ involving the amino acids that correspond to R538, E542 and H569 in colicin E7. ${ }^{4}$ However, H569 is bound to the $\mathrm{Zn}^{2+}$ ion and the change in its protonation state would be unfavourable.

Recently we have shown that the deletion of the N-terminal KRNK (446-449) sequence in NColE7 cancelled the catalytic activity ${ }^{21}$ in agreement with the previously demonstrated importance of the R447 residue. ${ }^{13}$ The requirement of cooperation of the N- and C-termini in NColE7 to exert its catalytic action may be developed into an allosteric activation control mechanism in a new artificial nuclease. ${ }^{22}$ This property would be an advantage over the artificial chimeric nucleases created by fusion of e.g. zinc-finger or TALE proteins and the FokI nuclease domain, ${ }^{23-25}$ where the allosteric control present in the native FokI ${ }^{26}$ is lost by exchanging its DNA binding domain with zinc-finger motifs. 
This may e.g. account for the known moderate cytotoxicity of zinc-finger nucleases designed for gene therapy. ${ }^{27}$ Therefore, it is important to understand the regulatory elements in artificial nucleases intended for in vivo applications. ${ }^{28}$

To better understand the role of the $\mathrm{N}$-terminal sequence

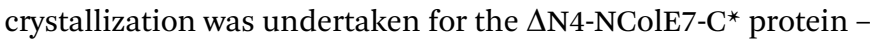
where the KRNK (446-449) sequence is replaced by a part of the GST purification tag lacking positively charged side chains. Previously its biochemical and biophysical properties were studied. ${ }^{21,29}$ Here we present the structure determination to $1.7 \AA$ resolution and a comparison to the previously published structures of NColE7. The possible influence of the mutation at the C-terminus was also elucidated by recloning the $\Delta$ N4-NColE7 gene. The NColE7-DNA complexes can only be crystallized with an inactive form of the protein. To obtain information on the local geometries of the active site, we performed also QM/MM calculations.

\section{Materials and methods}

\section{Construction of the genes of the mutant proteins}

The gene encoding the $\Delta \mathrm{N} 4$-NColE7 mutant was cloned from the pQE70 plasmid (a generous gift of Prof. K.-F. Chak, Institute of Biochemistry and Molecular Biology, National Yang Ming University, Taipei, Taiwan ${ }^{12}$ ) and inserted into the pGEX-6P-1 (GE Healthcare) vector between the EcoRI/XhoI restriction enzyme sites (E/X) as described earlier. ${ }^{21}$ The same fragment was also built in the pET-21a vector (Novagen) with the same restriction enzymes - $(\mathrm{E} / \mathrm{X}) \Delta \mathrm{N} 4-\mathrm{NColE} 7$ gene - and was recloned into the NdeI/XhoI sites $(\mathrm{N} / \mathrm{X})$ of the same vector: the $(\mathrm{N} / \mathrm{X}) \Delta \mathrm{N} 4-$ NColE7 gene. Since the insert DNA sequence contained a C-terminal stop codon, no C-terminal tags were expected to form. To check the effect of the C-terminal mutation, we have also cloned the gene of the NColE7-C* mutant into the pGEX-6P-1 vector.

\section{Protein expression and purification}

The expression and purification of the GST- $\Delta$ N4-NColE7-C* protein (for the protein sequences see Fig. 1.) and the cleavage of the glutathione S-transferase (GST) affinity tag at the N-terminus by the human rhinovirus C3 protease $^{30}$ was performed as described previously. ${ }^{29}$ The expression of the GST- $\Delta$ N4-NColE7, GST-NColE7-C* and (E/X) $\Delta$ N4-NColE7 proteins could not be carried out because of their toxicity to the host cell. On the other hand, the $(\mathrm{N} / \mathrm{X}) \Delta \mathrm{N} 4-\mathrm{NColE7}$ protein was successfully over expressed. The purification of this mutant was carried out using a Sepharose Fast Flow (GE Healthcare) ion exchange column equilibrated with phosphate-buffer (PBS: 0.14 M NaCl, $2.7 \mathrm{mM}$ $\left.\mathrm{KCl}, 10 \mathrm{mM} \mathrm{Na}_{2} \mathrm{HPO}_{4}, 1.8 \mathrm{mM} \mathrm{KH}_{2} \mathrm{PO}_{4}, \mathrm{pH}=7.7\right)$. The elution was carried out with a $\mathrm{NaCl}$ concentration gradient up to $1.0 \mathrm{M}$ through 15 column volume in the same buffer. The protein was loaded onto a Source 30S (GE Healthcare) column under the same conditions as mentioned above. The molecular mass of the purified protein was determined by ESI-MS as described by Czene et $a l^{21}$ The theoretical average mass of the holo protein $(\mathrm{N} / \mathrm{X}) \Delta \mathrm{N} 4-\mathrm{NColE} 7$ with its first Met removed and in complex with one $\mathrm{Zn}^{2+}$-ion is $14563.3 \mathrm{Da}$, in excellent agreement with the ESI-MS results of $14563.0 \mathrm{Da}$. This indicates that the purified protein was in its $\mathrm{Zn}^{2+}$-bound form.

\begin{tabular}{|c|c|}
\hline & \\
\hline (GST) $\Delta \mathrm{N} 4-\mathrm{NCOlE} 7-\mathrm{C}^{*}$ & - - - - - - GPLGSPEFPGKATGKGKPVNNKWLNNAGKDLGSPVPDRIANKLRDKEFKSFDDFRKKFWEEVSKDPEL \\
\hline (GST) $\Delta \mathrm{N} 4-\mathrm{NCOlE} 7$ & - - - - - -GPLGSPEFPGKATGKGKPVNNKWLNNAGKDLGSPVPDRIANKLRDKEFKSFDDFRKKFWEEVSKDPEL \\
\hline$(\mathrm{E} / \mathrm{X}) \Delta \mathrm{N} 4-\mathrm{NCOlE} 7$ & MASMTGGQQMGRGSEFPGKATGKGKPVNNKWLNNAGKDLGSPVPDRIANKLRDKEFKSFDDFRKKFWEEVSKDPEL \\
\hline$(\mathrm{N} / \mathrm{X}) \Delta \mathrm{N} 4-\mathrm{NCOlE} 7$ & MPGKATGKGKPVNNKWLNNAGKDLGSPVPDRIANKLRDKEFKSFDDFRKKFWEEVSKDPEL \\
\hline (GST) NCOlE7-C* & - - - GPLGSPEFKRNKPGKATGKGKPVNNKWLNNAGKDLGSPVPDRIANKLRDKEFKSFDDFRKKFWEEVSKDPEL \\
\hline Colicin E7 & KRNKPGKATGKGKPVNNKWLNNAGKDLGSPVPDRIANKLRDKEFKSFDDFRKKFWEEVSKDPEL \\
\hline Colicin E2 & KRNKPGKATGKGKPVGDKWLDDAGKDSGAPIPDRIADKLRDKEFKSFDD FRKKFWEEVSKDPEL \\
\hline Colicin E8 & KRNKPGKATGKGKPVGDKWLDDAGKDSGAP IPDRIADKLRDKEFKNFDDFRRKFWEEVSKDPEL \\
\hline Colicin E9 & KRNKPGKATGKGKPVGDKWLDDAGKDSGAPIPDRIADKLRDKEFKNFDDFRKKFWEEVSKDPDL \\
\hline Klebicin B & PRNMPGTASGKGQNVGNNWMGGTSTGDGAPVPSQIADKLRGKAFGSFDSFCRAFWKAVAADPDL \\
\hline s-type Pyocin & GRDLPGKVTGTGTDVEGSWLAGAGEGLGAPVPTRIADRLRDREFSSFDAFRRSFWQEVAADPEL \\
\hline Pyocin-S2 P. a & PRDVPGAATGKGQPVSGNWLGAASQGEGAPIPSQIADKLRGKTFKNWRDFREQFWIAVAND PEL \\
\hline TolA K. pneumoniae & PRNMPGTVSGKGQNVGNNWMGGASTGDGAPVPSQIADKLRGKTFGSFDSSRRAFWKAVADDSAL \\
\hline
\end{tabular}

576

(GST) $\triangle N 4$-NCOIE7-C* SKQFSRNNNDRMKVGKAPKTRTQDVSGKRTSFELHHEKPISQNGGVYDMDNISVVTPKRHIDIHQVNSSSGRIVTD

(GST) $\triangle N$ N - NCOIE7 SKQFSRNNNDRMKVGKAPKTRTQDVSGKRTSFELHHEKPISQNGGVYDMDNISVVTPKRHIDIHRGK

(E/X) $\triangle N$ N - NCOIE7 SKQFSRNNNDRMKVGKAPKTRTQDVSGKRTSEELHHEKPISONGGVYDMDNISVVTPKRHIDIHRGK

(N/X) $\Delta \mathrm{N} 4-\mathrm{NCOIE7}$ SKQFSRNNNDRMKVGKAPKTRTQDVSGKRTSFELHHEKPISQNGGVYDMDNISVVTPKRHIDIHRGK

(GST) NCOIE7-C* SKQFSRNNNDRMKVGKAPKTRTQDVSGKRTSFELHHEKPISQNGGVYDMDNISVVTPKRHIDIHQVNSSSGRIVTD

Colicin E7 SKOFSRNNNDRMKVGKAPKT RTODVSGKRTSFELHHEKPISONGGVYDMDNISVVTPKRHIDIHRGK

Colicin E2 SKQFKDSNKTNIQKGKAPFARKKDQVGGRERFELHHDKPISQDGGVYDMDNIRVTTPKRHIDIHRGK

Colicin E8 SKQFNPGNKKRLSQGLAPRARNKDTVGGRRSFE LHHDKPI SQDGGVYDMDNLRITTPKRHID IHRGQ

Colicin E9 SKQFKGSNKTNIQKGKAPFARKKDQVGGRERFELHHDKPISQDGGVYDMNNIRVTTPKRHIDIHRGK

Klebicin B SKOFYPDDIERMKLGRAPTVRFRDSVGKRVKVELHHKVEI SKGGDVYNVDNLNALTPKRH IE IHKGN

S-type Pyocin AGQFKKGNQGRMKKGLAPRVRELEQAGKRHSTELHHVDLISDGGEVYNIDNIHVVTPKQHVE IHSGK

Pyocin-S2 P. aer. SKQFNPGSLAVMRDGGAPYVRESEQAGGRIKIEIHHKVRIADGGGVYNMGNLVAVTPKRHIE IHKGGK

TOIA K. pneumoniae SKQFSEADINQMKAGRAPTADFLESVGKRVKIELHHEKEI SOGGAVMDVDNIKALTPKNH IETHKGK

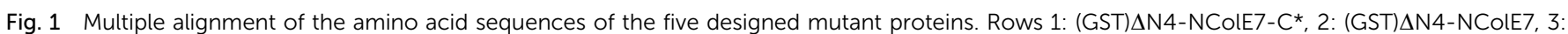

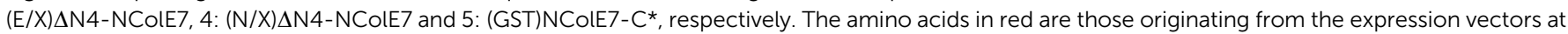

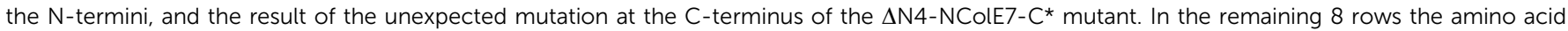

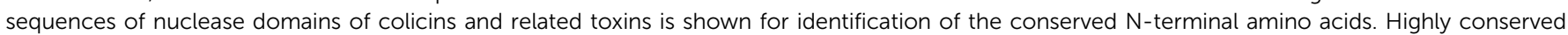
residues are in bold, while bold and italic means conserved amino acids. 


\section{Structure determination and refinement}

Details of the crystallization of $\Delta \mathrm{N} 4-\mathrm{NColE7-C} *$ and measurement of X-ray diffraction data have been provided earlier in ref. 29. The overall symmetry of the diffraction pattern corresponds to the Laue class $3 \mathrm{~m}$ and the systematically absent reflections are consistent with the space groups $P 3_{1} 21$ or $P 3_{2} 21$. The volume-to-mass ratio $V_{\mathbf{M}}$ of $1.99 \AA^{3} \mathrm{Da}^{-1}$ and the corresponding solvent content of $35 \%$ match well a structure with one molecule per asymmetric unit $\left(M_{\mathrm{r}} \sim 16.2 \mathrm{kDa}\right)$. It was possible to solve the structure by the Molecular Replacement method in the space group $P 3_{2} 21$ using MOLREP ${ }^{31}$ with the native NColE7 structure (PDB entry: $1 \mathrm{M}^{5} 8^{5}$ ) as a search model.

In the subsequent refinement of the structure, difficulties in modelling of the polypeptide chain were encountered in tracing the tagged $\mathrm{N}$-terminus of the protein. The refined model in the space group $P 3_{2} 21$, which had the lowest acceptable penalty score in $\mathrm{XDS}^{32}$ contained density close to the crystallographic twofold axis that could not be resolved into a meaningful chemical model and thus the space group published in ref. 29 was reconsidered. The N-terminus of the $\Delta \mathrm{N} 4-\mathrm{NColE7}-\mathrm{C}^{*}$ protein (Fig. 1) contains a GPLGSPEF additional sequence remaining after the cleavage of the GST tag during the protein expression and purification procedure. It was possible to trace the SPEF residues in this segment, found in a part of the crystal structure adjacent to the twofold axis. In the space group $P 3_{2} 21$ the first four (GPLG) residues would run into density of the same four residues of the symmetry related molecule (Fig. S1, ESI $\dagger$ ). Refinements in other possible space groups were therefore attempted. The structure could be refined in $P 3_{2}$ with a pseudo twofold axis replacing the crystallographic twofold axis in $P 3_{2} 21$. The refined model had a significantly lower $R_{\text {free }}$ and a comparable $R_{\text {work }}$ to the model obtained in $P 3_{2} 21$. In the space group $C 2$, which would correspond to three molecules per asymmetric unit one of them is related to another molecule in the unit cell by a crystallographic twofold axis giving rise to similar problems as in the $P 3_{2} 21$ space group. Due to the low redundancy refinement was not attempted in $P 1$. The results are summarized in Table 1.

After refinement with Refmac $5^{36}$ and manual rebuilding in COOT, ${ }^{37}$ it was possible to obtain a chemically sound model that contains all residues of the sequence from 446S (KRNK of WT NColE7 replaced by SPEF) to H573. Four N-terminal (442G, 443P, $444 \mathrm{~L}, 445 \mathrm{G}$, which is part of the GST tag) and $12 \mathrm{C}$-terminal residues (Q574-D585, this part of the sequence is indicated as the $\mathrm{C}^{*}$ mutations) were not modelled due to a disorder. The residual fragmented density close to the non-crystallographic twofold axis was modelled as water molecules. It was possible to trace the amino acids in the electron density up to those introduced by random mutation in the $\mathrm{C}$-terminus. Thus, the final structural model displays an intact $\beta \beta \alpha$ type metal binding site. In the refinement noncrystallographic symmetry (NCS) was not applied to allow for disruption of the twofold symmetry that relates the two crystallographical independent molecules. The difference and omit maps revealed the positions of the $\mathrm{Zn}^{2+}$-ion. Residual density was modelled as water molecules if they fulfilled the expected geometrical conditions. Furthermore, nine sulfate, two chloride and two acetate ions were modelled in the difference electron density. The almost perfect twofold symmetry that relates the two molecules also with respect to the overall $B$-values, $27.3 \AA^{2}$ and $27.1 \AA^{2}$ for the A and B molecule, respectively, is not completely maintained in the position of the ions vide infra.

\section{QM/MM calculations on protein-DNA complexes}

The protein setup was performed based on the crystal structures $3 \mathrm{FBD}^{10}$ and $1 \mathrm{M}^{10} 8^{5}$ as well as the construction of the QM/MM

Table 1 Data processing in the three different space groups (details on the data collection experiment are given in ref. 29). The resolution is reduced from 1.6 to $1.7 \AA$ in the refinement, to exclude poorly merging weak reflections in this range. $R_{\text {merge }}=\sum_{h k l} \sum_{i}\left|I_{i}(h k l)-\langle I(h k l)\rangle\right| / \sum_{h k l} \sum_{i} I_{i}(h k l)$, where $l_{i}(h k l)$ is the intensity measurement for a given reflection and $l_{i}(h k l)_{i}$ is the average intensity for multiple measurements of this reflection. The values in parentheses correspond to the highest resolution shell

\begin{tabular}{|c|c|c|c|}
\hline Space group & $P 3_{2}$ & $P 3_{2} 21$ & $C 2$ \\
\hline Resolution range/Å & $40-1.7(1.8-1.7)$ & $40-1.7(1.8-1.7)$ & $40-1.7(1.8-1.7)$ \\
\hline Unit-cell parameters $a, b, c(\AA)$ & $55.4,55.4,73.2$ & $55.4,55.4,73.2$ & $96.0,55.4,73.2$ \\
\hline$\alpha, \beta, \gamma\left({ }^{\circ}\right)$ & $90,90,120$ & $90,90,120$ & $90,90.0,90$ \\
\hline Total reflections & $154648(24128)$ & $154714(24240)$ & $153962(24038)$ \\
\hline Unique reflections & $53902(8489)$ & $27529(4395)$ & $78323(12217)$ \\
\hline Average multiplicity/redundancy & $2.9(2.8)$ & $5.6(5.5)$ & $2.0(2.0)$ \\
\hline Completeness (\%) & $97.3(93.0)$ & $99.4(97.6)$ & $94.2(90.6)$ \\
\hline$\langle I / \sigma(I)\rangle$ & $15.7(2.7)$ & $22.8(3.9)$ & $15.2(2.4)$ \\
\hline$R_{\text {merge }}(\%)$ & $4.1(39.3)$ & $4.4(43.6)$ & $3.2(36.8)$ \\
\hline Number of molecules per asymmetric unit & 2 & 1 & 3 \\
\hline $\begin{array}{l}\text { Refinement } R_{\text {work }} / R_{\text {free }} \\
\text { R.m.s. deviation from ideality }{ }^{a}\end{array}$ & $0.200(0.290) / 0.234(0.331)$ & $0.191(0.260) / 0.277(0.320)$ & $0.210(0.230) / 0.250(0.300)$ \\
\hline Bonds/Å & 0.01 & & \\
\hline Angles $/^{\circ}$ & 1.28 & & \\
\hline Number of atoms (protein) & 4179 & & \\
\hline Number of water molecules & 104 & & \\
\hline Number of bound $\mathrm{Zn}^{2+}$-ions & 2 & & \\
\hline Number of atoms in ions ( 2 acetates, 9 sulfates, 2 chlorides) & 55 & & \\
\hline Ramachandran plot outliers ${ }^{b} / \%$ & 0.8 & & \\
\hline
\end{tabular}

${ }^{a}$ With respect to Engh and Huber parameters. ${ }^{33}{ }^{b}$ The three Ramachandran outliers ${ }^{34,35}$ N461, L465 and D471 had well defined backbone density for the modelled conformations in both molecules. 
system, following the recommended protocol ${ }^{38,39}$ that is almost identical to those used in our previous QM/MM studies. ${ }^{40-42}$ The quantum system (System 1) consisted of 169 atoms for NColE7 and 156 for $\Delta \mathrm{N} 4-\mathrm{NColE7}$ (Fig. S2, ESI $\dagger$ ) including the $\mathrm{Zn}^{2+}$-ion, $\mathrm{H} 544, \mathrm{H} 545$, H569, H573, V555 and the side chain of R447 (only for NColE7). The MM part was divided into System 2 (surrounding of the quantum region) that is allowed to move in the QM/MM minimizations and System 3 (the rest of the protein) that is kept fixed.

The QM/MM calculations were carried out by employing a modified version of the ComQum program. ${ }^{38,39,43}$ The Turbomole 6.3 program $^{44}$ was used for the quantum chemical calculations carried out using DFT method and using PerdewBurke-Ernzerhof (PBE) functional ${ }^{45}$ and Ahlrich's def2-SVP basis set. $^{46}$ Resolution of the identity (density-fitting) was used to expedite the DFT calculations. MM calculations were carried out in the AMBER 8 program package (sander module) and the ff $03^{47,48}$ force field (parm99 set in Amber).

Further details are deposited in the ESI $\dagger$ (see chapters S1-S3 and references therein).

\section{PDB code}

The coordinates of the $\Delta \mathrm{N} 4-\mathrm{NColE7}-\mathrm{C}^{*}$ refined in space group $P 3_{2}$ have been deposited in the PDB with the reference: 3ZFK.

\section{Results}

\section{Description of the structure of the $\Delta \mathrm{N} 4-N C o l E 7-C^{*}$ protein}

The two protein chains in the final model are virtually identical, the superimposition of their backbones gives an rmsd (root mean square deviation) of $0.05 \AA$ A (Fig. S3, ESI $\dagger$ ). It is noteworthy that the four residues of fused GST-tag that have replaced the four deleted residues (KRNK) in the intact NColE7 sequence are visible in the electron density. The close interactions between the N-termini of the A and B molecules illustrated in Fig. 2a involve only the first four amino acids of the tag. Mass spectra and N-terminal analysis have verified that the protein is intact. However, in the C-terminus only the residues that are part of the NColE7 sequence are visible in the electron density. Inspection of the structure shows that there is sufficient space in the crystal packing to accommodate the last 12 residues in disordered conformations.

Electron densities interpreted as chloride ions are located in similar positions on the surface of the two molecules, their neighbors are G455 and three water molecules, all in the expected hydrogen bond distances. Fig. 2a shows how the molecules related by the non-crystallographic twofold axes are linked by sulfate ions that neutralize positively charged side chains. One of these (marked in red) is located on the noncrystallographic twofold axis. Two of the sulfate ions serve as ligands to the bound $\mathrm{Zn}^{2+}$-ions. The sulfate ions associated with the molecules A and B conform with the overall twofold symmetry that relates the two molecules. This is not the case for the acetate ions. The two acetate ions that were identified in the electron density are both bound to molecule B, where they form hydrogen

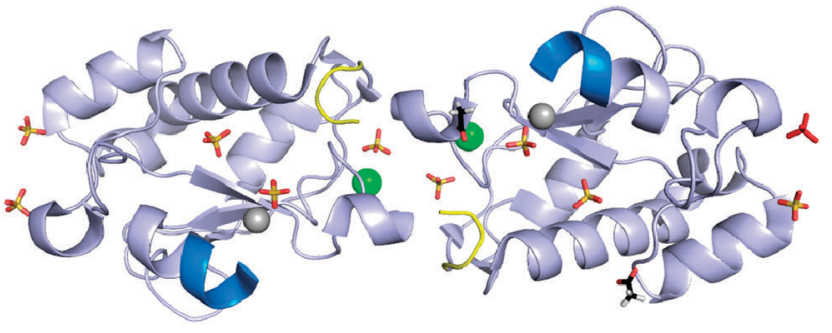

(a)

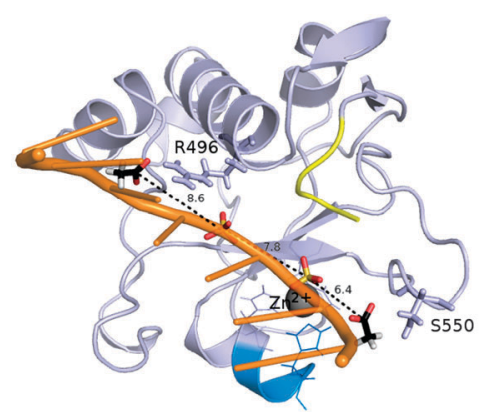

(b)

Fig. 2 (a) The two independent molecules $A$ and $B$ of $\triangle$ N4-NColE7-C* with bound ions. Molecule $A$ is in the left of the figure, and molecule $B$ to the right. Their $\mathrm{N}$-termini are shown in yellow and the $\mathrm{C}$-termini in bright blue. $\mathrm{Zn}^{2+}$-ions are illustrated as grey spheres, chloride ions as green spheres; sulfate and acetate ions are shown in a stick representation. The sulfate ion drawn in red bound to molecule $B$ is a crystallographically related match of the sulfate ion in the top left bound to molecule $A$. (b) Molecule B with its bound anions. The side chains of the hydrogen bond partners of the acetate ions are shown in a stick representation. The two acetate ions (bound to the labeled residues) and two sulfate ions close to the active site of molecule $B$ are bound similarly to the phosphodiester groups of one of the DNA chains in the structure $2 \mathrm{IVH}^{8}$

bonds with R496 and S550. It is worth mentioning that the anions bound to the B molecule nicely mimic the predicted binding sites of the DNA phosphodiester groups (Fig. 2b).

Some distinct interactions involving the amino acids of the truncated $\mathrm{N}$-terminus could be identified in the crystal structure

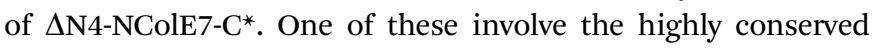
W464 located in a hydrophobic pocket formed by the residues of the N-terminal loop (L465, P475, V476, P477), the central part of the protein (F499, W500, L509, F513) and the HNH motif (V563) as shown in Fig. 3 a and b. Residues from the $\mathrm{N}$-terminus interact with the HNH loop and with one of the $\beta$-sheets within the C-terminal HNH motif as shown in Fig. 3c. These interactions make the N-terminal loop fill the space (Fig. 3d) between the $\mathrm{HNH}$ motif and the central part of the protein including nonspecific DNA and Im7 binding helices.

These interactions - most of them found in the other known NColE7 structures - may stabilize the active site. This is supported by our recent studies on the $\mathrm{HNH}$ motif itself, ${ }^{49}$ on the $\Delta \mathrm{N} 25$-NColE7 mutant lacking the major part of the N-terminal loop, ${ }^{21}$ as well as on the NColE7 triple mutant including T454A, K458A and W464A mutations. ${ }^{50}$

Fig. 4 shows the environment of the $\mathrm{Zn}^{2+}$-ions bound in the two molecules, A and B. Both are coordinated by three histidine 


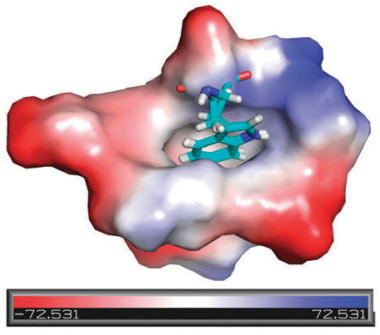

(a)

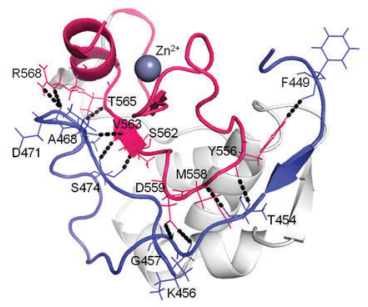

(c)

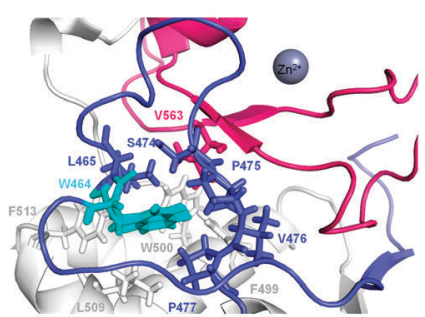

(b)

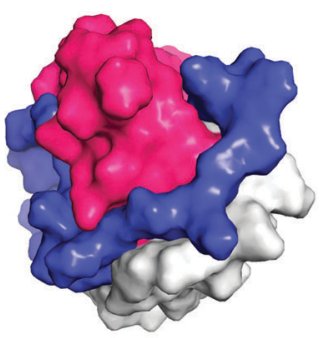

(d)
Fig. 3 Interactions of the $\mathrm{N}$-terminal loop. (a) The vacuum electrostatic surface of the surroundings of W464. (b) The hydrophobic environment around W464. (c) The identification of possible hydrogen bonds formed between the amino acids of the $\mathrm{N}$-terminal loop and those of the $\mathrm{HNH}$ motif. (d) Shape complementarity of the $\mathrm{N}$-terminal loop between the $\mathrm{C}$-terminal $\mathrm{HNH}$ motif and the central parts of the protein (grey). In (b), (c) and (d) the $\mathrm{N}$-terminus is colored in blue, while the $\mathrm{HNH}$ motif is red.

residues and a sulfate ion. The C-terminal $\mathrm{H} 573$ is poorly defined in both molecules. The refined $B$-value for the $\mathrm{Zn}^{2+}$ ion is significantly higher in molecule A than in molecule B, 40.54 and 24.57 , respectively. The excess residual density at the position of the $\mathrm{Zn}^{2+}$-ion in molecule A could indicate a disorder that has not been sufficiently well modeled. The closest water molecule to $\mathrm{Zn}^{2+}$ is W2072, in a distance of $4.0 \AA$. The $B$-value of

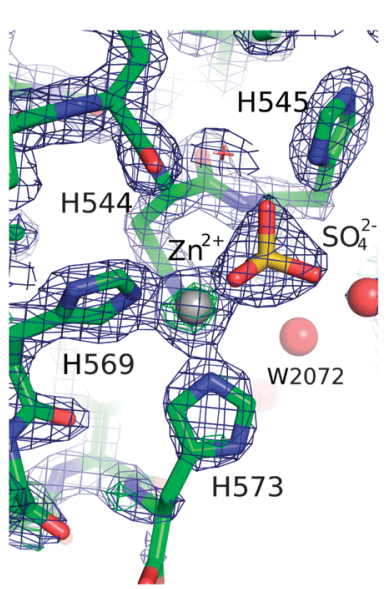

(a)

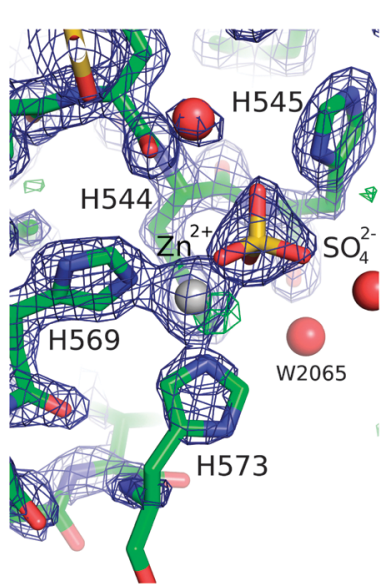

(b)
Fig. 4 The surroundings of $\mathrm{Zn}^{2+}$-ion in the active site of the two molecules. A Sigma-A weighted $2 F_{\text {obs }}-F_{\text {calc }}$ electron density map at 1.5 sigma $\left(0.48 \mathrm{e}^{-} \AA^{-3)}\right.$ is shown in blue and $F_{\text {obs }}-F_{\text {calc }}$ difference map electron density at 3.0 sigma cut-off $\left(0.37 \mathrm{e}^{-} \AA^{-3}\right)$ in green and red, respectively for the (a) A and (b) B molecule in the crystal structure of $\triangle N 4-N C o l E 7-C^{*}$. The peak in the difference density $1.25 \AA$ away from the $\mathrm{Zn}^{2+}$-ion was not modelled. the $\mathrm{Zn}^{2+}$-ion in molecule $\mathrm{B}$ is significantly lower and residual density is found $1.25 \AA$ away from the $\mathrm{Zn}^{2+}$-ion. Furthermore a water molecule (W2065) is located in the second coordination sphere of $\mathrm{Zn}^{2+}$, at a distance of $3.9 \AA$.

\section{Computational results: QM/MM calculations - comparison of} the catalytic centre from the calculations and crystal structures

QM/MM calculations provide a valuable complementary method for the local structural details of proteins e.g., in ref. 51 and 52, QM/MM calculations were performed for the $\Delta \mathrm{N} 4-\mathrm{NColE7}-\mathrm{Zn}^{2+}$-DNA and the active NColE7- $\mathrm{Zn}^{2+}$-DNA model structures. We expected that these calculations should predict the active state of the protein complexed with $\mathrm{Zn}^{2+}$ and DNA, and give important atomic details (position of hydrogen atoms, protonation states, etc.).

As can be seen in Fig. 5a, the overall optimized structure of the two proteins is similar whereas small structural differences are confined to the active centre. These differences are seen in the orientations of the metal coordinating histidines, notably H544 and the position of the metal ion in $\Delta \mathrm{N} 4-\mathrm{NColE} 7$. The bond angles to ligands in the coordination sphere of the metal ion are more significantly influenced than the bond lengths. Surprisingly, in both QM/MM optimized structures the $\mathrm{Zn}^{2+}$-ion in the active centre became pentacoordinated in a slightly distorted trigonal bipyramidal geometry (Fig. 5b).

The deletion of the N-terminal KRNK residues likely causes changes also in key steps of the catalysis. The supposed general base $\mathrm{H} 545$ is tilted in the modelled $\Delta \mathrm{N} 4$-NColE7 structure (Fig. 6a), as compared to the optimized NColE7-DNA complex structure. The catalytic water molecule activated by this residue is in different orientation in the two optimized structures. When R447 is present, it serves as a hydrogen bond donor to this water molecule in the optimized structure.

Changes in the active site of NColE7 affected the substrate DNA chain. One of the two bases at the scissile phosphate (CYT11) is tilted in the NColE7-DNA complex (Fig. 6a) and is no longer coplanar with its guanine pair. This strain in DNA may facilitate its hydrolytic cleavage. In contrast, in the $\triangle$ N4-NColE7-DNA optimized structure the CYT11 is nearly coplanar to its pair on the opposite strand. We also noted a difference in the $\mathrm{P}-\mathrm{O}^{\prime}{ }^{\prime}$ scissile bond lengths in the optimized structures (Fig. 6b): the increased length in the presence of NColE7 suggests higher probability of the cleavage by the WT enzyme compared to the $\Delta$ N4-NColE7 mutant.

\section{Discussion}

\section{Comparison of the $\triangle \mathrm{N} 4-\mathrm{NColE7-C} *$ crystal structure with other available NColE7 structures}

Fig. 7a shows that the mutations at the $\mathrm{N}$ - and C-termini did not influence significantly the $\Delta \mathrm{N} 4$-NColE7 fragment (residues 450-573) of the structure compared to NColE7 (PDB entry: $1 \mathrm{MZ}^{6}$ ) the all-atom rmsd being $0.23 \AA$ A. Accordingly, the lack of DNase activity is not due to conformational changes but it is directly related to the mutations. The only clear difference was

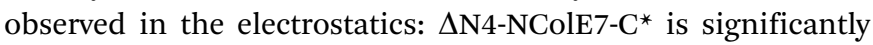
less positive than NColE7 (Fig. S4, ESI $\dagger$ ). 


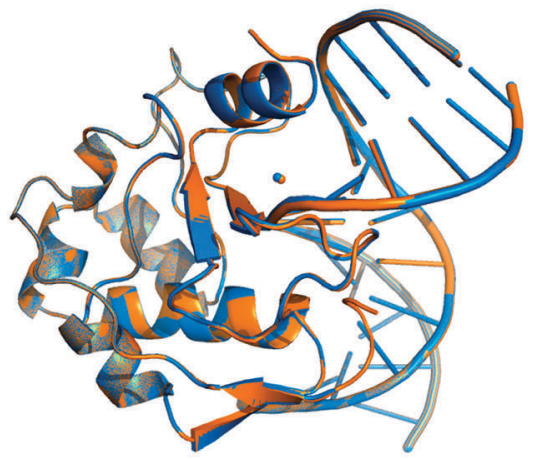

(a)

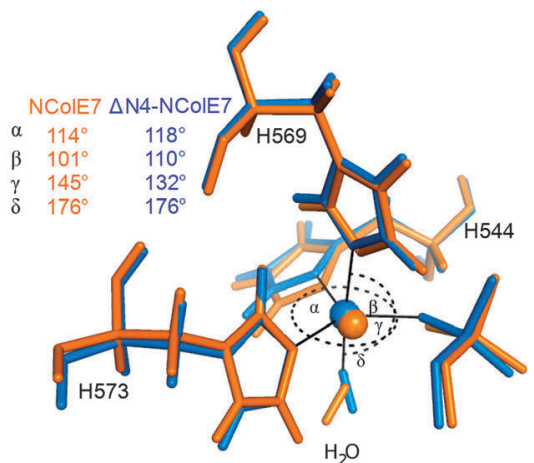

(b)

Fig. 5 (a) The superimposed structures of NColE7-DNA (orange) and $\triangle$ N4-NColE7-DNA (blue) complexes, both optimized using the QM/MM method. (b) The active centre of the corresponding structures: the $\mathrm{Zn}^{2+}$-ion is pentacoordinated with three His residues, one water molecule and an oxygen atom of the scissile phosphate in its coordination sphere.

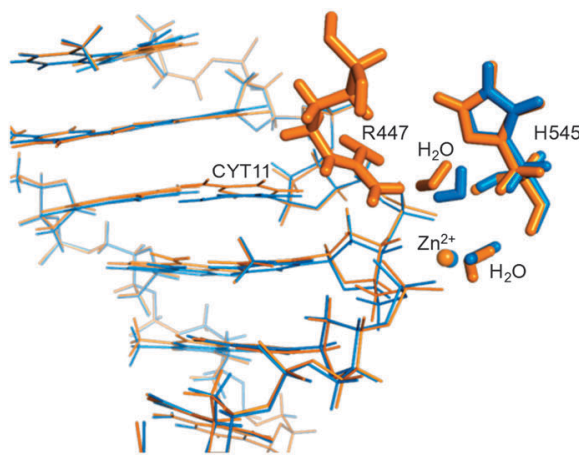

(a)

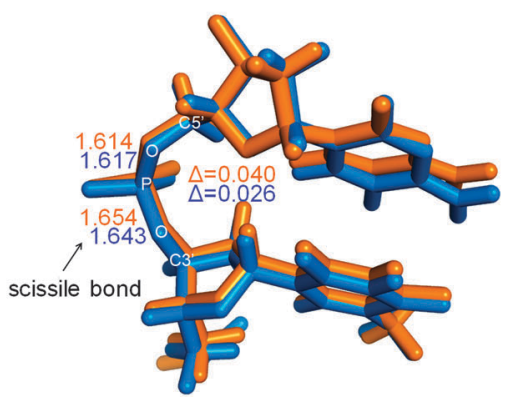

(b)

Fig. 6 (a) DNA binding of NColE7 (orange) and $\triangle$ N4-NColE7 (blue) in the QM/MM optimized structures. Major differences appear in the position of the $\mathrm{H} 545$ and its hydrogen-bonded water molecule and in the position of the plane of CYT11. (b) P-O bond lengths within the scissile phosphodiester bond in the same structures.

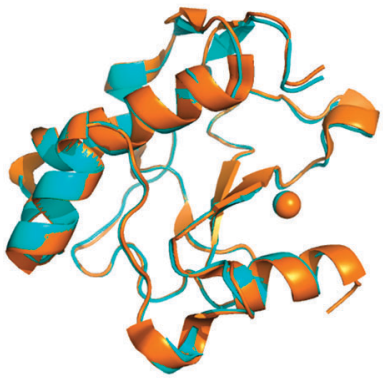

(a)

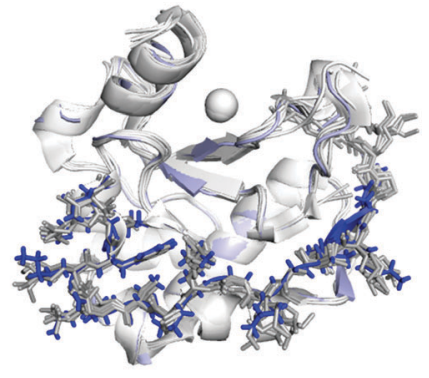

(b)
Fig. 7 (a) Structural alignment based on the identical amino acids of the $\triangle N 4-N C O l E 7-C^{*}$ (blue; PDB code: 3ZFK) and WT NColE7 (orange; PDB code: $1 M Z 8^{6}$ ) shows good agreement between the structures of the two proteins. (b) Structural alignment of the $\mathrm{N}$-terminal loops in selected NColE7 domains including the PDB structures $1 \mathrm{MO} 8{ }^{5}{ }^{5} 1 \mathrm{MZ},{ }^{6}{ }^{6} 1 \mathrm{PT} 3,{ }^{3}$ $1 Z \mathrm{NS}^{7}{ }^{7} 1 \mathrm{ZNV},{ }^{7} 7 \mathrm{CEI},{ }^{4} 2 \mathrm{IVH}^{8}{ }^{8} 2 \mathrm{JAZ}^{9}{ }^{9} 2 \mathrm{JBO}^{9},{ }^{9} 2 \mathrm{JBG}^{9}$ and $3 \mathrm{FBD}^{10}$ all in light grey and the $\triangle 4-N C O L E-C *$ in blue.

The structure and relative position of the N-terminus are not affected by DNA or Im7 binding in NColE7 variant crystal structures. $^{3-10}$ It also remained unchanged in the $\Delta$ N4-NColE7-C* protein upon the mutation of the KRNK sequence at the $\mathrm{N}$-terminus as Fig. 7b shows. This confirms that additional interactions exist between the N-terminal loop and the rest of the protein, which are responsible for keeping the positive charges at the $\mathrm{N}$-terminus close to the catalytic centre.

In $\Delta \mathrm{N} 4-\mathrm{NColE7-} \mathrm{C}^{*}$ it was also possible to identify all the hydrogen bonds formed by the conserved N560 with the backbone atoms of E546, K547, G557 and D557 in the flexible HNH loop (Fig. S5, ESI $\dagger$ ). ${ }^{9}$ N560 has a high impact on the catalytic activity, since it fixes the position of the general base H545. 9,13 The position of the four active site histidines is well preserved in most of the NColE7 structures. The only exceptions are the DNA complexes of the H545 mutated inactive enzyme (1ZNS, ${ }^{7}$ $\left.2 \mathrm{IVH}^{8}\right)$ - where one or more His side-chains are tilted (Fig. 8a). Similarly, the rotation of the $\mathrm{H} 573$ side-chain imidazole ring by $\sim 17$ degrees is observed in the active site of $\Delta$ N4-NColE7-C* relative to the corresponding imidazole in the 1M08 NColE7 structure.

A slight shift in the position is seen for the $\mathrm{Zn}^{2+}$-ion and the substrate mimicking sulfate ion in $\Delta \mathrm{N} 4-\mathrm{NColE7- \textrm {C } ^ { * }}$ relative to the $\mathrm{Zn}^{2+}$ containing NColE7 structures in Fig. 8b. This did not affect the strong metal ion binding of $\Delta$ N4-NColE7-C ${ }^{*}{ }^{21}$ 

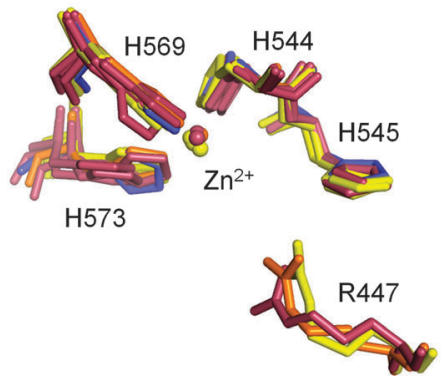

(a)

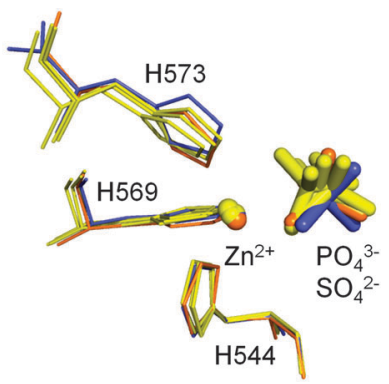

(b)

Fig. 8 (a) Comparison of the arrangement of the metal ion and coordinating histidine residues in the active site of $\triangle N 4-N C O E F-C^{*}$ (in blue) with other NColE7 structures (NColE7 in orange: $1 \mathrm{MO}^{\circ}{ }^{5} \mathrm{NColE7/Im7}$ in yellow: $1 \mathrm{MZ8},{ }^{6}$ 1ZNV, ${ }^{7} 7 \mathrm{CEI},{ }^{4} 2 \mathrm{JAZ},{ }^{9} 2 \mathrm{JBO},{ }^{9} 2 \mathrm{JBG}^{9}{ }^{9}$ NColE7-DNA in brown: $1 \mathrm{PT}^{3},{ }^{3} 2 \mathrm{IVH}^{8}$, $\left.1 Z \mathrm{ZS}^{7}{ }^{7} 3 \mathrm{FBD}^{10}\right)$. (b) Orientation of the phosphate ions $(1 \mathrm{MO} 8,1 \mathrm{MZ} 8,2 \mathrm{JAZ})$ and the sulfate ions (2JBG and the $\triangle \mathrm{N} 4-\mathrm{NCO} E 7-\mathrm{C}^{\star}$ ) applying the same color coding.

By contrast $\Delta \mathrm{N} 25-\mathrm{NColE7}$ and T454A/K458A/W464A-NColE7 mutants with modified $\mathrm{N}$-terminal sequences showed impaired $\mathrm{Zn}^{2+}$-binding ability due to the structural changes of the active centre. ${ }^{21,50}$ Consequently, we suggest that the interactions between the N-terminus and the $\mathrm{HNH}$ motif are essential for the mutual stabilization of their structure and for the proper folding of the catalytic centre.

The comparison of the $\Delta \mathrm{N} 4-\mathrm{NColE7}-\mathrm{C}^{*}$ structure and other published crystal structures to the QM/MM computational models of NColE7-DNA and $\triangle$ N4-NColE7-DNA can give a deeper insight into the catalytic differences in the active centre. While the $\mathrm{Zn}^{2+}$-coordination is similar in the compared structures, as shown by $\mathrm{Zn}^{2+}$-donor atom distances listed in Table 2, the difference between the $\mathrm{P}-\mathrm{O}\left(3^{\prime}\right)$ and $\mathrm{P}-\mathrm{O}\left(5^{\prime}\right)$ bond lengths of the scissile phosphate is only significant in the optimized active structure, but not in the crystal structures. It must be emphasized that all the experimental results were obtained for inactive forms of the protein, i.e. either the metal ion was absent or a functional residue was mutated. This difference between the computed NColE7-DNA and $\triangle$ N4-NColE7-DNA complexes may indicate the slight changes in the active centre that finally contribute to the loss of nuclease activity upon deletion of the $\mathrm{N}$-terminal positively charged residues.

The $\mathrm{Zn}^{2+}$-ion and the positively charged $\mathrm{N}$-terminal amino acids in the active site - new possible roles in the catalytic function

In the crystal structure of $\Delta \mathrm{N} 4-\mathrm{NColE} 7-\mathrm{C}^{*}$ the B molecule had a $\mathrm{Zn}^{2+}$-binding site with significant residual density in the vicinity, at a difference map peak of $1.25 \AA$, as shown in Fig. 2. The major difference lies not in the protein monomers, but in the metal ions: $\mathrm{Zn}^{2+}$-ion $\mathrm{B} 402$ is less rigid and with a significantly lower $(62 \%)$ atomic displacement parameter than that of the $\mathrm{Zn}^{2+}$-ion in the $\mathrm{A}$ molecule. The metal-ligand coordination is essentially the same in both molecules, but our modelling studies hinted at the coordination of an additional water molecule (Fig. 4). This is in agreement with the results of the QM/MM calculations. In the optimized structures (Fig. 6a) a water molecule that was weakly bound $(R(\mathrm{Zn}-\mathrm{O})=$ $3.7 \AA)$ in the starting structure approached the metal ion to $1.9 \AA$ and $2.0 \AA$ for $\triangle$ N4-NColE7 and NColE7, respectively. Such water molecules may be activated during the catalytic process to serve either as a general acid or base. This model is consistent with an artificial nuclease system ${ }^{53,54}$ and a similar type of coordination was observed experimentally in an NColE9 crystal structure $\left(1 \mathrm{FSJ}^{55}\right)$. It is worth mentioning that in the crystal structure of $\Delta \mathrm{N} 4-\mathrm{NColE} 7-\mathrm{C}^{*} \mathrm{H}_{2} \mathrm{O}(2072)$ in the A molecule and $\mathrm{H}_{2} \mathrm{O}(2065)$ in the B molecule are located $\sim 4.0 \AA$ from the $\mathrm{Zn}^{2+}$-ion.

Sequence comparison of related colicin and pyocin bacterial toxins showed that the arginine corresponding to R447 in colicin E7 is highly conserved (Fig. 1). Crystal structures of $\mathrm{HNH}$ nucleases showed that an arginine stretches into the active site e.g. R447 in NColE7, ${ }^{5,6,10}$ R5 in NColE9, ${ }^{56}$ R99 in Vvn, ${ }^{16} \mathrm{R} 57$ in Sm endonuclease ${ }^{57}$ and R93 in nuclease A. ${ }^{58}$ The distance from the side chain of this arginine to the metal ion located in the active centre is $\sim 6-7 \AA$ in the presence of DNA or a phosphate/sulfate ion situated between the positively charged residues, and $>10 \AA$ in the absence of it. Based on Vvn crystal structures it was hypothesized that the arginine side chain binds and stabilizes the cleaved DNA to decelerate the reverse reaction. ${ }^{16,17}$ In $\mathrm{NColE9}^{20}$ and in Sm endonuclease ${ }^{59}$ related HNH nucleases the arginine corresponding to R447 in NColE7 was proposed to stabilize the pentavalent transition state. The R447A mutation in NColE7 reduced the in vitro DNase activity to $\sim 15 \%$ of the initial value ${ }^{13}$ supposed to be due to the decreased DNA binding affinity. In contrast, we have recently shown that the mutations of the positive charges within the KRNK (446-449) sequence do not significantly affect the strength of the DNA binding. ${ }^{60}$ This study also demonstrated that the positively charged lysines can partially replace the missing arginine in its function.

Surprisingly, precise location of R447 in the presence of the DNA has only been determined in the 18bp DNA-D493Q

Table 2 Comparison of the NColE7-DNA crystal structures with the QM/MM optimized structures and the 3ZFK structure. Bond lengths in $\AA$

\begin{tabular}{|c|c|c|c|c|c|c|c|c|}
\hline Structures & $\mathrm{Zn}^{2+}-\mathrm{O}(\mathrm{DNA})$ & $\mathrm{Zn}^{2+}-\mathrm{H} 544$ & $\mathrm{Zn}^{2+}-\mathrm{H} 569$ & $\mathrm{Zn}^{2+}-\mathrm{H} 573$ & $\mathrm{P}-\mathrm{O}\left(3^{\prime}\right)$ & $\mathrm{P}-\mathrm{O}\left(5^{\prime}\right)$ & $\Delta \mathrm{P}-\mathrm{O}\left(3^{\prime}-5^{\prime}\right)$ & Ref. \\
\hline 1PT3 (without $\mathrm{Zn}^{2+}$ ) & - & - & - & - & 1.607 & 1.588 & 0.019 & 3 \\
\hline 3FBD (without $\mathrm{Zn}^{2+}$ ) & - & - & - & - & 1.616 & 1.594 & 0.022 & 10 \\
\hline 2IVH (H545Q) & 1.769 & 2.039 & 2.136 & 1.837 & 1.604 & 1.596 & 0.008 & 8 \\
\hline 1ZNS (H545E) & 2.017 & 1.798 & 1.941 & 2.047 & 1.591 & 1.579 & 0.012 & 7 \\
\hline 3ZFK (without DNA) A & - & 1.977 & 2.187 & 2.287 & - & - & - & This work \\
\hline 3ZFK (without DNA) B & - & 1.672 & 2.073 & 2.090 & - & - & - & This work \\
\hline$\Delta \mathrm{N} 4-\mathrm{NColE7-DNA}$ calc. & 2.003 & 2.081 & 2.147 & 2.044 & 1.643 & 1.617 & 0.026 & This work \\
\hline NColE7-DNA calc. & 2.067 & 2.095 & 2.148 & 2.117 & 1.654 & 1.614 & 0.040 & This work \\
\hline
\end{tabular}


NColE7 mutant complex, ${ }^{10}$ and it was not possible to locate it in the three other crystal structures of DNA complexes. ${ }^{3,7,8}$ In this complex R447 clearly interacts with the phosphodiester group of the DNA molecule. In spite of the close contacts, this residue has unusually high temperature factors 60-70 $\AA^{2}$ compared to those of the rest of the molecule (Fig. S6, ESI $\dagger$ ) indicating its high flexibility. The experiments on the cytotoxic behavior of the mutants (see later) also demonstrated that even the location of the positively charged amino acid residue in the amino acid sequence close to the $\mathrm{N}$-terminus is not strictly determined.

Considering the above observations we can not exclude that the flexible arginine side-chain mediates the proton transfer in NColE7. After protonating the leaving group it may become instantly re-protonated by $\mathrm{H} 545$. These two residues get close (Fig. 9) to each other and a water-mediated hydrogen bond is formed between them during the catalytic cycle. The initial source of the proton on $\mathrm{H} 545$ is the water molecule, which has to become deprotonated to perform the nucleophilic attack at the partially positively charged phosphorus atom. This would be in agreement with the recently proposed shuttle mechanism according to which the leaving group is protonated by the hydrogen ion originating from the same water molecule that initiated the nucleophilic attack. ${ }^{61}$

The above mechanism would be similar to the one found in a serine recombinase-mediated DNA cleavage. ${ }^{62}$ Although arginine is rarely mentioned in the literature to behave as an acid but the environment of an enzyme active site can significantly shift the $\mathrm{p} K_{\mathrm{a}}$ values of critical residues. It can be considered plausible in biological systems, ${ }^{63}$ especially in the presence of a basic leaving group as it is the $3^{\prime}$-alcoholate ion. This would provide a reasonable answer to the still unsolved but intriguing question about the identity of the general acid that protonates the leaving group in NColE7. It shall also be mentioned that the multiple roles of $\mathrm{R} 447$ can be partially

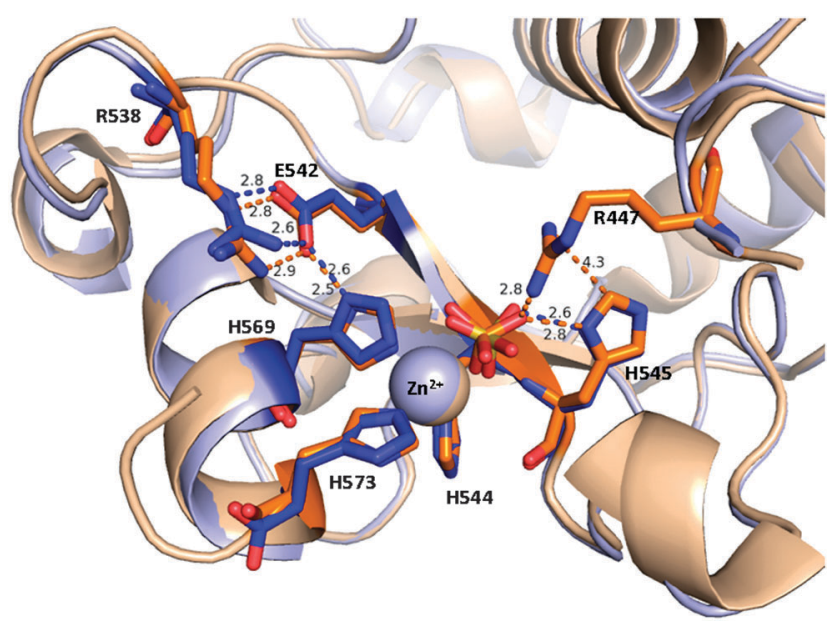

Fig. 9 Short interatomic distances reflecting possible hydrogen bonds in

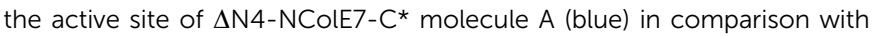
NColE7 (PDB: 1M08, in orange). The hydrogen bonds among R538, E542 and $\mathrm{H} 569$ (the $\mathrm{H} 569$ residue also binds the $\mathrm{Zn}^{2+}$-ion) form a putative proton channel. replaced by other positive charges, such as lysine side-chains or the $\mathrm{N}$-terminal amino group in a suitable position.

\section{The effect of the $\mathrm{N}$ - and C-terminal mutations on the cytotoxicity}

Toxic variants of NColE7 kill the bacterial cell through their nuclease activity already during the cloning process due to the minor level of expression in the applied cloning systems. ${ }^{22,64}$ Taking advantage of this we can obtain information on the catalytic activity of an NColE7 mutant already in the early stage of the experiments. At the same time, the minuscule probability to obtain a gene of erroneous sequence, resulting in an inactive mutant, is increased. These mutations may reveal the necessity of each amino acid residue and may affect the protein structure, metal ion or DNA binding or the catalytic process itself. This event led to a C-terminal modification resulting in extra 9 amino acids within the inactive (GST) $\Delta$ N4-NColE7-C* protein. This points to the fact that the expected GST- $\Delta$ N4-NColE7 protein was cytotoxic for the $E$. coli cells.

To understand this and to investigate the impact of the mutations at the two termini of the GST- $\Delta$ N4-NColE7-C* protein we have recloned the $\Delta$ N4-NColE7 gene into the pET-21a vector as described in Materials and methods (the expected sequences are depicted in Fig. 1). While the (E/X) $\Delta$ N4-NColE7 variant was cytotoxic, the $(\mathrm{N} / \mathrm{X}) \Delta \mathrm{N} 4-\mathrm{NColE} 7$ protein of correct sequence was successfully overexpressed in E. coli as a $\mathrm{Zn}^{2+}$-bound protein (the ESI-MS proof of the metal ion binding is shown in Fig. S7, ESI $\dagger$ ) similarly to the GST- $\Delta$ N4-NColE7-C* mutant. The latter protein was proven to bind both $\mathrm{Zn}^{2+}$-ions and DNA in vitro strongly, ${ }^{21}$ thus it is the lack of another feature that allows for expression without cytotoxic effects.

The data collected in Table 3 show that the $\Delta$ N4-NColE7 with an arginine close to the $\mathrm{N}$-terminus in a similar position to the WT NColE7 is cytotoxic. The only exception is the active GST$\Delta \mathrm{N} 4$-NColE7 nuclease. However, in this protein the GST fusion tag carries several positive charges both on the surface and in the linker region (Fig. S8, ESI $\dagger$ ). One of these may allow for the catalytic process in GST- $\Delta$ N4-NColE7, but not in GST- $\Delta$ N4NColE7-C* ${ }^{*}$ The access of the bulky GST tag to the active site is most probably prohibited by the newly formed C-terminus in the latter protein. In parallel to this the same $C^{*}$ mutation can not

Table 3 The correlation of the cytotoxic activity with the modifications at the $\mathrm{N}$ - and $\mathrm{C}$-termini of the NColE7 in different mutants in this study

\begin{tabular}{llll}
\hline & $\begin{array}{l}\text { Position of R near } \\
\text { the N-terminus }\end{array}$ & $\begin{array}{l}\text { C-terminal } \\
\text { modification }\end{array}$ & Cytotoxicity \\
\hline NColE7 & 447 & - & + \\
GST- $\Delta$ N4-NColE7-C & $-{ }^{a}$ & + & - \\
GST- $\Delta$ N4-NColE7 & $-{ }^{a}$ & - & + \\
(E/X) $\Delta$ N4-NColE7 & $445^{b}$ & - & + \\
(N/X) $\Delta$ N4-NColE7 & - & - & - \\
GST-NColE7-C & 447 & + & +
\end{tabular}

${ }^{a}$ There is no positively charged residue within a distance of 10 amino acids of the original position of the R447. ${ }^{b}$ The gene of $\triangle$ N4-NColE7 was inserted into a plasmid that added a short sequence containing the arginine at position R445. 
prevent the GST-NColE7- $\mathrm{C}^{*}$ to be cytotoxic, providing further proof of the importance of the KRNK sequence.

\section{Conclusions}

The truncated mutant of NColE7 nuclease, lacking the positively charged KRNK amino acid string at the end of the N-terminal sequence proved to be catalytically inactive. The presence of the $\mathrm{N}$-terminal loop - distant in the sequence from the catalytic centre - playing an intramolecular allosteric role in the enzymatic process, as found in NColE7, is a fascinating phenomenon to study. This feature of NColE7 may be applied in the engineering of controlled artificial nucleases. The crystal structure of the $\triangle$ N4-NColE7-C* mutant, determined at $1.7 \AA$ A resolution revealed direct information about the structural role of the remaining $\mathrm{N}$-terminal sequence, and it also provided indirect information on the role of the deleted KRNK (446-449) string. The additional C-terminal modification was shown to control the enzymatic activity by preventing the distal positively charged amino acids on bulky chains from approaching the catalytic site.

As the structure of $\Delta \mathrm{N} 4-\mathrm{NColE7}-\mathrm{C}^{*}$ does not significantly differ from the structures determined so far for NColE7 variants we can conclude that the lack of its catalytic activity is not due to a change in the overall structure. The extensive interactions between the unchanged part of the N-terminal loop and the rest of the protein are not influenced by the deletion of the KRNK amino acid sequence. These interactions may contribute to the mutual stabilization of the N-terminal loop and the catalytic centre.

The cytotoxic activity of the designed mutant proteins indicated that it can be associated with a positively charged residue close to the N-terminus. R447 could not be modeled in the majority of NColE7 variant crystal structures, which indicates a great flexibility. It leads us to propose that R447 beside the previously suggested functions may also behave as a flexible general acid that assist in the transfer of the proton from the general base to the leaving group. According to the QM/MM calculations the presence of R447 also facilitates the distortion of the substrate DNA chain promoting the hydrolysis of the scissile phosphodiester group. QM/MM calculations pointed out that in the active NColE7-DNA complex - that cannot be studied by crystallographic methods due to the catalytic reaction - the bond length of the scissile bond in contrast to the crystal structures of inactive variants is elongated, and the plane of the nucleobase at the cleavage site is tilted. These phenomena were not observed in the optimized $\Delta \mathrm{N} 4$-NColE7 structure.

Both the QM/MM calculations and the careful inspection of the crystal structure revealed the possibility of the coordination of a water molecule to the $\mathrm{Zn}^{2+}$-ion as the fifth ligand. Such a metal ion activated water molecule may participate in the catalytic process as a nucleophilic reagent after passing its proton to an amino acid side-chain of the protein, or as a general acid providing its proton in the final step of the reaction.

Our results showed that the mechanism of action of NColE7 requires some revisions. The essential catalytic role of the positively charged amino acids (such as R447 in the WT enzyme), as well as the presence of a water molecule as the fifth ligand in the $\mathrm{Zn}^{2+}$ coordination sphere has to be considered. The detailed description of the particular role of these residues will be possible upon further combined experimental and computational studies.

\section{Acknowledgements}

Financial support from the Hungarian Scientific Research Found (OTKA-NKTH CK80850), TÁMOP-4.2.2/B-10/1-2010-0012, TÁMOP 4.2.4.A/2-11-1-2012-0001 'National Excellence Program' and JSPS is greatly acknowledged. E.T. and A.C. thank Danish Ministry of Science, Innovation \& Higher Education for the fellowships provided. L.R. acknowledges the support from the Grant Agency of the Czech Republic (grant 1431419S).

\section{References}

1 R. D. Finn, J. Tate, J. Mistry, P. C. Coggill, S. J. Sammut, H. R. Hotz, G. Ceric, K. Forslund, S. R. Eddy, E. L. Sonnhammer and A. Bateman, Nucleic Acids Res., 2008, 36, D281-D288.

2 A. Veluchamy, S. Mary, V. Acharya, P. Mehta, T. Deva and S. Krishnaswamy, Bioinformation, 2009, 4, 80-83.

3 K. Hsia, K. Chak, P. Liang, Y. Cheng, W. Ku and H. S. Yuan, Structure, 2004, 12, 205-214.

4 T. Ko, C. Liao, W. Ku, K. Chak and H. S. Yuan, Structure, 1999, 7, 91-102.

5 Y. Cheng, K. Hsia, L. G. Doudeva, K. Chak and H. S. Yuan, J. Mol. Biol., 2002, 324, 227-236.

6 M. Sui, L. Tsai, K. Hsia, L. Doudeva, W. Ku, G. Han and H. Yuan, Protein Sci., 2002, 11, 2947-2957.

7 L. Doudeva, D. Huang, K. Hsia, Z. Shi, C. Li, Y. Shen, Y. Cheng and H. Yuan, Protein Sci., 2006, 15, 269-280.

8 Y. Wang, W. Yang, C. Li, L. G. Doudeva and H. S. Yuan, Nucleic Acids Res., 2007, 35, 584-594.

9 H. Huang and H. S. Yuan, J. Mol. Biol., 2007, 368, 812-821. 10 Y.-T. Wang, J. D. Wright, L. G. Doudeva, H.-C. Jhang, C. Lim and H. S. Yuan, J. Am. Chem. Soc., 2009, 131, 17345-17353.

11 K. B. Levin, O. Dym, S. Albeck, S. Magdassi, A. H. Keeble, C. Kleanthous and D. S. Tawfik, Nat. Struct. Mol. Biol., 2009, 16, 1049-1055.

12 K. Chak, W. Kuo, F. Lu and R. James, J. Gen. Microbiol., 1991, 137, 91-100.

13 Z. Shi, K. Chak and H. Yuan, J. Biol. Chem., 2005, 280, 24663-24668.

14 J. H. Eastberg, J. Eklund, R. Monnat Jr. and B. L. Stoddard, Biochemistry, 2007, 46, 7215-7225.

15 R. H. van den Heuvel, S. Gato, C. Versluis, P. Gerbaux, C. Kleanthous and A. J. Heck, Nucleic Acids Res., 2005, 33, e96.

16 C. L. Li, L. I. Hor, Z. F. Chang, L. C. Tsai, W. Z. Yang and H. S. Yuan, EMBO J., 2003, 22, 4014-4025. 
17 K. Hsia, C. Li and H. Yuan, Curr. Opin. Struct. Biol., 2005, 15, 126-134.

18 C. Garinot-Schneider, A. J. Pommer, G. R. Moore, C. Kleanthous and R. James, J. Mol. Biol., 1996, 260, 731-742.

19 A. J. Pommer, S. Cal, A. H. Keeble, D. Walker, S. J. Evans, U. C. Kühlmann, A. Cooper, B. A. Connolly, A. M. Hemmings, G. R. Moore, R. James and C. Kleanthous, J. Mol. Biol., 2001, 314, 735-749.

20 D. C. Walker, T. Georgiou, A. J. Pommer, D. Walker, G. R. Moore, C. Kleanthous and R. James, Nucleic Acids Res., 2002, 30, 3225-3234.

21 A. Czene, E. Németh, I. G. Zóka, N. I. Jakab-Simon, T. Körtvélyesi, K. Nagata, H. E. M. Christensen and B. Gyurcsik, J. Biol. Inorg. Chem., 2013, 18, 309-321.

22 E. Németh, G. K. Schilli, G. Nagy, C. Hasenhindl, B. Gyurcsik and C. Oostenbrink, J. Comput.-Aided Mol. Des., 2014, 28, 841-850.

23 Y. G. Kim, J. Cha and S. Chandrasegaran, Proc. Natl. Acad. Sci. U. S. A., 1996, 93, 1150-1160.

24 J. K. Joung and J. D. Sander, Nat. Rev. Mol. Cell Biol., 2013, 14, 49-55.

25 M. Christian, T. Cermak, E. L. Doyle, C. Schmidt, F. Zhang, A. Hummel, A. J. Bogdanove and D. F. Voytas, Genetics, 2010, 186, 757-761.

26 D. A. Wah, J. A. Hirsch, L. F. Dorner, I. Schildkraut and A. K. Aggarwal, Nature, 1997, 388, 97-100.

27 T. I. Cornu and T. Cathomen, Methods Mol. Biol., 2010, 649, 237-245.

28 B. Gyurcsik and A. Czene, Future Med. Chem., 2011, 3, 1935-1966.

29 A. Czene, E. Tóth, B. Gyurcsik, H. Otten, J. N. Poulsen, L. Lo Leggio, S. Larsen, H. E. M. Christensen and K. Nagata, Acta Crystallogr., Sect. F: Struct. Biol. Cryst. Commun., 2013, 69, 551-554.

30 P. Walker, L. Leong, P. NG, S. Tan, S. Waller, D. Murphy and A. Porter, Bio/Technology, 1994, 12, 601-605.

31 A. Vagin and A. Teplyakov, Acta Crystallogr., Sect. D: Biol. Crystallogr., 2010, 66, 22-25.

32 W. Kabsch, Acta Crystallogr., Sect. D: Biol. Crystallogr., 2010, 66, 133-144.

33 R. A. Engh and R. Huber, Acta Crystallogr., Sect. A: Found. Crystallogr., 1991, 47, 392-400.

34 R. A. Laskowski, M. W. MacArthur, D. S. Moss and J. M. Thornton, J. Appl. Crystallogr., 1993, 26, 283-291.

35 V. B. Chen, W. B. Arendall 3rd, J. J. Headd, D. A. Keedy, R. M. Immormino, G. J. Kapral, L. W. Murray, J. S. Richardson and D. C. Richardson, Acta Crystallogr., Sect. D: Biol. Crystallogr., 2010, 66, 12-21.

36 G. N. Murshudov, A. A. Vagin and E. J. Dodson, Acta Crystallogr., Sect. D: Biol. Crystallogr., 1997, 53, 240-255.

37 P. Emsley, B. Lohkamp, W. G. Scott and K. Cowtan, Acta Crystallogr., Sect. D: Biol. Crystallogr., 2010, 66, 486-501.

38 U. Ryde, J. Comput.-Aided Mol. Des., 1996, 10, 153-164.

39 U. Ryde and M. H. M. Olsson, Int. J. Quantum Chem., 2001, 81, 335-347.
40 L. Rulisek, E. I. Solomon and U. Ryde, Inorg. Chem., 2005, 44, 5612-5628.

41 M. Srnec, F. Aquilante, U. Ryde and L. Rulisek, J. Phys. Chem. B, 2009, 113, 6074-6086.

42 V. Klusak, C. Barinka, A. Plechanovova, P. Mlcochova, J. Konvalinka, L. Rulisek and J. Lubkowski, Biochemistry, 2009, 48, 4126-4138.

43 T. A. Rokob and L. Rulísek, J. Comput. Chem., 2012, 33, 1197-1206.

44 O. Treutler and R. Ahlrichs, J. Chem. Phys., 1995, 102, 346-354. 45 J. P. Perdew, K. Burke and M. Ernzerhof, Phys. Rev. Lett., 1996, 77, 3865-3868.

46 A. Schäfer, H. Horn and R. Ahlrichs, J. Chem. Phys., 1992, 97, 2571-2577.

47 Y. Duan, C. Wu, S. Chowdhury, M. C. Lee, G. Xiong, W. Zhang, R. Yang, P. Cieplak, R. Luo, T. Lee, J. Caldwell, J. Wang and P. Kollman, J. Comput. Chem., 2003, 24, 1999-2012.

48 M. C. Lee and Y. Duan, Proteins, 2004, 55, 620-634.

49 B. Gyurcsik, A. Czene, H. Jankovics, N. I. Jakab-Simon, K. Ślaska-Kiss, A. Kiss and Z. Kele, Protein Expression Purif., 2013, 89, 210-218.

50 E. Németh, T. Körtvélyesi, M. Kožíšek, P. W. Thulstrup, H. E. M. Christensen, M. N. Asaka, K. Nagata and B. Gyurcsik, J. Biol. Inorg. Chem., 2014, DOI: 10.1007/s00775-014-1186-6.

51 U. Ryde and K. Nilsson, J. Am. Chem. Soc., 2003, 125, 14232-14233.

52 L. Rulisek and U. Ryde, J. Phys. Chem. B, 2006, 110, 11511-11518.

53 X. Sheng, X. Guo, X. M. Lu, G. Y. Lu, Y. Shao, F. Liu and Q. Xu, Bioconjugate Chem., 2008, 19, 490-498.

54 X. Sheng, X. M. Lu, J. J. Zhang, Y. T. Chen, G. Y. Lu, Y. Shao, F. Liu and Q. Xu, J. Org. Chem., 2007, 72, 1799-1802.

55 U. C. Kühlmann, A. J. Pommer, G. R. Moore, R. James and C. Kleanthous, J. Mol. Biol., 2000, 301, 1163-1178.

56 M. J. Maté and C. Kleanthous, J. Biol. Chem., 2004, 279, 34763-34769.

57 S. V. Shlyapnikov, V. V. Lunin, M. Perbandt, K. M. Polyakov, V. Y. Lunin, V. M. Levdikov, C. Betzel and A. M. Mikhailov, Acta Crystallogr., Sect. D: Biol. Crystallogr., 2000, 56, 567-572.

58 M. Ghosh, G. Meiss, A. Pingoud, R. E. London and L. C. Pedersen, J. Biol. Chem., 2005, 280, 27990-27997.

59 P. Friedhoff, I. Franke, G. Meiss, W. Wende, K. L. Krause and A. Pingoud, Nat. Struct. Biol., 1999, 6, 112-113.

60 E. Németh, T. Körtvélyesi, P. W. Thulstrup, H. E. M. Christensen, M. Kozísek, K. Nagata, A. Czene and B. Gyurcsik, Protein Sci., 2014, 23, 1113-1122.

61 J. A. Bueren-Calabuig, C. Coderch, E. Rico, A. Jimenez-Ruiz and F. Gago, ChemBioChem, 2011, 12, 2615-2622.

62 R. A. Keenholtz, K. W. Mouw, M. R. Boocock, N. S. Li, J. A. Piccirilli and P. A. Rice, J. Biol. Chem., 2013, 288, 29206-29214.

63 P. J. Silva, C. Schulz, D. Jahn, M. Jahn and M. J. Ramos, J. Phys. Chem. B, 2010, 114, 8994-9001.

64 L. C. Anthony, H. Suzuki and M. Filutowicz, J. Microbiol. Methods, 2004, 58, 243-250. 